\title{
Distributed Power Control Algorithm in Orthogonal Frequency Division Multiple Cognitive Radio Systems for Fading Channel
}

\author{
https://doi.org/10.3991/ijoe.v14i03.8416 \\ Lingling Chen $(\bowtie)$ \\ Jilin Institute of Chemical Technology, Jilin, Jilin, China \\ Jilin University, Changchun, Jilin, China \\ cl1807900@ 163.com \\ Qi Feng, Xingquan Gao, Huipeng Wang, Jiwen Liang \\ Jilin Institute of Chemical Technology, Jilin, Jilin, China
}

\begin{abstract}
In order to guarantee the quality of service (QoS) for primary users (PUs) and secondary users (SUs) in fading channel, a distributed power control algorithm is proposed based on convex optimization theory in underlay OFDM cognitive radio networks (CRNs). Our purpose obtains the maximum transmit data rate of each SU at all subcarriers under three constraints of the maximum allowable transmission power, the minimum signal to interference plus ratio (SINR) of each SU and the maximum allowable interference generated from SUs to PU at each subcarrier. Simulation results show that the performance of the proposed algorithms $(\mathrm{m} 2)$ are superior to the geometric programming algorithm (m1) in fading channel environment.
\end{abstract}

Keywords-Cognitive radio networks, distributed power control algorithm, convex optimization theory

\section{Introduction}

As the explosive growth of wireless communication services and applications, the limited spectrum resources becomes increasingly crowded and have not satisfied the growing needs of people [1]. In order to dynamically allocate and share spectrum, cognitive radio (CR) [2] is proposed to improve the efficiency of spectrum utilization. Currently, the underlay spectrum sharing mode is most effective where multiple SUs and PUs can use spectrum resource at the same time and satisfy the constraint of the interference temperature (IT) threshold [3]. Thus, we focus on underlay CRNs [4-5] with multiple PUs and multiple SUs to study resource allocation problem.

In CRNs, the distributed power control algorithm can improve the transmission efficiency, reduce message exchange and computational burdens, which is one of the key technologies in resource allocation [6-10]. In [6], a distributed power control algorithm based on a cooperative game theoretic is proposed which satisfied the interference power constraint to protect transmission QoS of PUs. An interference-aware 
radio resource allocation based orthogonal frequency division multiple access (OFDMA) in cognitive radio networks is proposed to aim at maximizing secondary users throughput under the interference constraint of PUs in [7]. In [8], it consider the power efficiency optimization problem in the cognitive radio (CR) networks under both the average packet delay constraint of each CR transmitter (CR-Tx) and the interference constraint at primary receiver (PU-Rx). The authors propose a cooperative approach to make each CR-Tx know the interference level at the PU-Rx when there is no central control node in the network and no assistant sensors are deployed to do the interference measuring jobs. In [9], the resource allocation problem, which aims to maximize the weighted sum rate in orthogonal frequency division multiplexing based (OFDM-based) multi-cell cognitive radio networks (CRNs) with multiple description coding multi-cast (MDCM), is investigated. Network-wide optimization of transmit power with the goal of maximizing the total throughput, promises significant system capacity gains in interference-limited data networks, which was proposed in [10]. A large number of the aforementioned distributed power control algorithm are proposed based on perfect channel, and they may be not suitable to actual CR communication environment without taking into the near-far effect on the QoS of PUs and the minimum SINR at SU account.

Based on the above discuss, in this paper, a distributed power control algorithm is proposed to minimum the interference power from SU-Tx to PU-Rx based on convex optimization theory in underlay OFDM CRNs. In order to guarantee communication quality of PUs and SUs in fading channel, our proposed power control scheme not only considers that the transmit power of each SU at all subcarriers should not exceed its maximum power, but also takes the minimum SINR at secondary receivers (SUTxs) and the interference temperature threshold in each subcarrier into account. The proposed algorithm is well adapt to practical dynamic communication environment. Simulation results show that the proposed algorithm is superior to algorithms $(\mathrm{m} 2)$ are superior to the geometric programming algorithm (m1) [11] in fading channel environment.

\section{System model and problem formulation}

In this section, we consider $T$ primary users (PUs) and $M$ secondary users (SUs) in OFDM cognitive network with $K$ subcarriers in underlay scenario.

Owing to the battery capacity, the transmit power of each SU must be lower than is not above its maximum transmit power, i.e.,

$$
\sum_{k=1}^{K} p_{k}^{i} \leq P_{\max }^{i}
$$

where $P_{\max }^{i}$ is the threshold of the maximum power of the secondary user $i$ in all subcarriers.

In order to make sure the QoS of the PUs and consider near-far effect and reduce exchanging message of the SUs, the interference power generated from each SU in 
each subcarriers is lower than the bearable threshold of the PU. Thus, the weighting interference temperature is introduced. It can be defined as follows

$$
p_{k}^{i} h_{k}^{i t} \leq \omega_{k}^{i t} I_{k}^{t}
$$

where $h_{k}^{i t}$ is the interference gain from the PU $t$ to the SU $i$ at the subcarriers $k$. $I_{k}^{t}$ presents the maximum interference power level of subcarrier $k . \omega_{k}^{i t}$ is the weighting factor, which is proportional to the distance between the SU $i$ and PU $t$ at subcarrier $k$.

In order to guarantee the reliable QoS of the SUs the signal to interference noise ratio (SINR) at each SU-Rx should exceed the threshold

$$
\gamma_{\min }^{i} \leq \gamma_{k}^{i}
$$

where $\gamma_{\min }^{i}\left(\gamma_{\min }^{i}>0\right)$ is the minimum required SINR value at the $i$ SU. $\gamma_{k}^{i}$ denotes the SINR at the $i$ SU in the $k$ subcarrier.

Our optimization objective is to maximize the transmit data rate of each SU at all subcarriers, and simultaneously meet constraints (1), (2) and (3). Thus, the optimization problem can be depicted as

$$
\begin{aligned}
& \max \sum_{k=1}^{K} \log _{2}\left(1+\gamma_{k}^{i}\right) \\
& \text { s.t. }\left\{\begin{array}{l}
\sum_{k=1}^{K} p_{k}^{i} \leq P_{\max }^{i} \\
p_{k}^{i} \geq 0, \forall k \in\{1,2, \mathrm{~L}, K\}, i \in\{1,2, \mathrm{~L}, M\} \\
\gamma_{k}^{i} \geq \gamma_{\min }^{i} \\
p_{k}^{i} h_{k}^{i t} \leq \omega_{k}^{i t} I_{k}^{t}
\end{array}\right.
\end{aligned}
$$

where $\gamma_{k}^{i}=\frac{g_{k}^{i i} p_{k}^{i}}{N_{k}+h_{k}^{i t} T_{k}^{t}} . N_{k}$ represents the background noise at the $k$ subcarrier. $g_{k}^{i i}$ is the direct channel gain at the $k$ subcarrier. $T_{k}^{t}$ denotes the PU $t$ 's transmit power at the subcarriers $k$.

Obviously, the optimization (4) is a nonlinear programming [12] which is a concave maximization problem. The problem (4) can be converted to a convex function as follows. 


$$
\begin{aligned}
& \min -\sum_{k=1}^{K} \log _{2}\left(1+\gamma_{k}^{i}\right) \\
& \text { s.t. }\left\{\begin{array}{l}
\sum_{k=1}^{K} p_{k}^{i} \leq P_{\max }^{i} \\
p_{k}^{i} \geq 0, \forall k \in\{1,2, \mathrm{~L}, K\}, i \in\{1,2, \mathrm{~L}, M\} \\
\log _{2}\left(1+\gamma_{k}^{i}\right) \geq \log _{2}\left(1+\gamma_{\min }^{i}\right) \\
p_{k}^{i} h_{k}^{i t} / \omega_{k}^{i t} \leq I_{k}^{t}
\end{array}\right.
\end{aligned}
$$

\section{Distributed power control algorithm}

In this section, the distributed power control algorithm is applied to solve the transmit data rate problem of each SU on all subcarriers in OFDM CRNs. The distributed power control algorithm reveals the hidden convexity structure which can be resolved by Lagrange dual principle [13]. Therefore, the Lagrange function of (5) can be given by

$$
\begin{aligned}
& L(p, \xi, \lambda)=-\sum_{k=1}^{K} \log _{2}\left(1+\gamma_{k}^{i}\right)+\xi\left(\sum_{k=1}^{K} p_{k}^{i}-P_{\max }^{i}\right) \\
& +\sum_{k} \chi_{k}\left[\log _{2}\left(1+\gamma_{k}^{i}\right)-\log _{2}\left(1+\gamma_{\min }^{i}\right)\right]+\sum_{k} \lambda_{k}\left(p_{k}^{i} h_{k}^{i t} / \omega_{k}^{i t}-I_{k}^{t}\right)
\end{aligned}
$$

where $P=\left[p_{1}, \mathrm{~L}, p_{M}\right]^{T}$ is the transmission power vector. $\xi=\left[\xi_{1}, \xi_{2}, \mathrm{~L}, \xi_{M}\right]^{T} \geq 0$ denotes vector of dual variable. $\lambda_{k}$ represents a Lagrange multiplier.

According to the dual decomposition theory, the Lagrange dual function for (6) is givenby

$$
D(\xi, \lambda)=\sum_{k} \min _{p} L_{k}(p, \xi, \lambda)+\left(\xi p_{\max }^{i}-\sum_{k} \chi_{k} \log _{2}\left(1+\gamma_{\min }^{i}\right)-\sum_{k} \lambda_{k} I_{k}^{i}\right)
$$

where

$$
L_{k}(p, \xi, \lambda)=\left(\chi_{k}-1\right) \log _{2}\left(1+\gamma_{k}^{i}\right)+\xi p_{k}^{i}+\lambda_{k} p_{k}^{i} h_{k}^{i t} / \omega_{k}^{i t}
$$

And, the dual problem (6) is given as

$$
\min D(\xi, \lambda)
$$

$$
\text { subject to : } \xi \geq 0, \lambda \geq 0
$$

According to the KKT condition [14], the optimal transmit power of each SU at each subcarrier can be obtained by the following equation

$$
\frac{L_{k}(p, \xi, \lambda)}{\partial p_{k}^{i}}=0
$$


And the solution is

$$
p_{k}^{i}=\frac{\omega_{k}^{i t}\left(1+\chi_{k}\right)}{\omega_{k}^{i t} \xi+\lambda_{k} h_{k}^{i t}}-\frac{N_{k}+h_{k}^{i j} T_{k}^{j}}{g_{k}^{i}}
$$

The above problem can be solved by using subgradient method. Thus, we construct the subgradient iteration algorithm to update the Lagrange multipliers as follows

$$
\begin{gathered}
\xi(t+1)=\max \left(0, \xi(t)+\phi\left[P_{\max }^{i}-\sum_{k=1}^{K} p_{k}^{i}\right]\right)^{+} \\
\chi_{k}(t+1)=\max \left(0, \chi_{k}(t)+v\left[\log _{2}\left(1+\gamma_{k}^{i}\right)-\log _{2}\left(1+\gamma_{\min }^{i}\right)\right]\right)^{+} \\
\lambda_{k}(t+1)=\max \left(0, \lambda_{k}(t)+\varphi\left(p_{k}^{i} h_{k}^{i t} / \omega_{k}^{i t}-I_{k}^{t}\right)\right)^{+}
\end{gathered}
$$

where $[X]^{+}=\max \{0, X\}, t$ represents the iteration time and $\phi, v, \varphi$ are step sizes. The step sizes and multipliers $\xi, \chi_{k}$ and $\lambda_{k}[15]$ should be carefully chosen to ensure the fast convergence rate.

The distributed power control algorithm is below:

Step 1: Initialization: Set $t=0, \xi(0)>0, \chi_{k}(0)>0, \lambda_{k}(0)>0, \gamma_{i}^{\min }>0$, $0 \leq p_{k}^{i}(0) \leq p_{\max }^{i}, \phi>0, v>0, \varphi>0$.

Step 2: Calculate at the SU-Rx of link $i$ : Compute the sum of interference and noise power $N_{k}+h_{k}^{i j} T_{k}^{j}$. Update Lagrange multiplier $\xi$ by (12). Feedback information $N_{k}+h_{k}^{i j} T_{k}^{j}$ and $\xi$ to the SU-Tx of link $i$.

Step 3: Calculate at the SU-Tx of link $i$ : Receive $N_{k}+h_{k}^{i j} T_{k}^{j}$ and $\xi$, respectively. Update Lagrange multiplier $\chi_{k}$ and $\lambda_{k}$ by (13) and (14).

Step 4: Calculate the transmit power value of SU-Tx by (10).

Step 5: Go to step 2.

\section{Simulation results}

In this section, the computer simulations are presented to demonstrate the performance of the distributed power control algorithm(m2) by comparison with Geometric Programming algorithm(m1) [11].

In the underlay network, we suppose that there are 3 active cognitive users and 8 subcarriers in OFDMA primary systems, and the bandwidth of each subcarrier is 62.5 $\mathrm{kHz}$. The initial transmission power vector is randomly selected from interval 
$(0,0.01) \mathrm{mW}$. The power budgets $P_{\max }^{i}$ and the background noise levels $N_{k}$ are randomly chosen from the intervals $(0.9,1.1)$ and $(0.01,0.10)$ respectively. The simulation results are shown from Figure 1 to Figure 5.

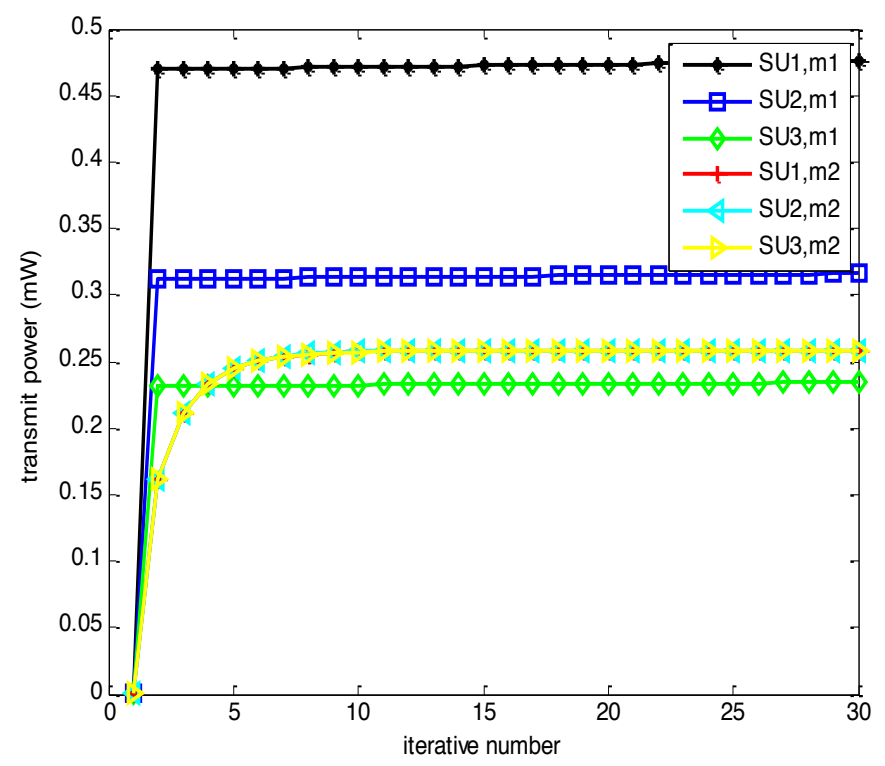

Fig. 1. The SINR of each SU

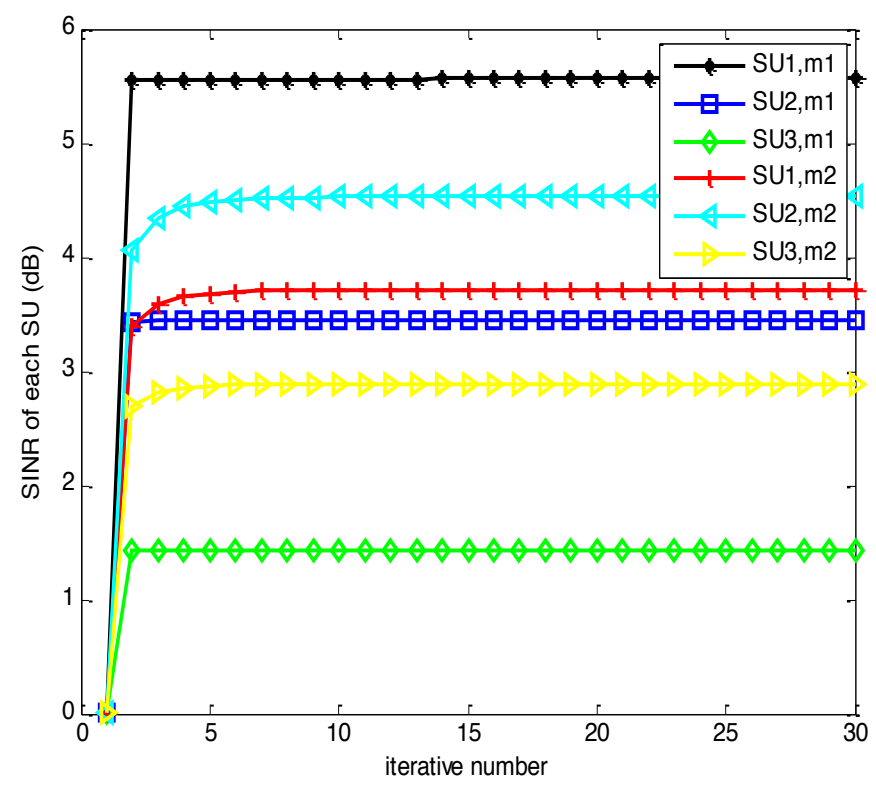

Fig. 2. The transmit power of each SU 
Fig. 1 and Fig. 2 present the transmit power and SINR of each SU for $\mathrm{m} 1$ and $\mathrm{m} 2$. We can clearly see that each SU satisfies the constrain of maximize allowable transmitting power and the minimum SINR in $\mathrm{m} 1$ and $\mathrm{m} 2$. Through comparing with $\mathrm{m} 1$ and $\mathrm{m} 2$, the transmit power and SINR are larger of SU1 for $\mathrm{m} 1$ than that of the proposed algorithm $\mathrm{m} 2$. However, the total throughput of $\mathrm{m} 2$ is higher than $16.7 \%$ that of the $\mathrm{m} 1$ in the fading channel. The numerical simulation is given from Fig. 3

According to the principle of the total interference generated from SUs to PU, we can see that $\mathrm{m} 2$ is significantly smaller than $\mathrm{m} 1$ from Fig. 4 . That means $\mathrm{m} 2$ has a lower badly effect on PU, which may affect QoS of primary user network.

In Fig.5, we analyze the system performance of the energy under multiple PUs and SUs in underlay CRNs. As expected, the total energy consumption of $\mathrm{m} 2$ is less than $30 \%$ in $\mathrm{m} 1$. Thus, we can save a lot of energy in $\mathrm{m} 2$, which can improve energy efficiency.

From the above graphs, we can draw a conclusion that the performances of the proposed $\mathrm{m} 2$ algorithm is much superior to that of $\mathrm{m} 1$ given in [11].

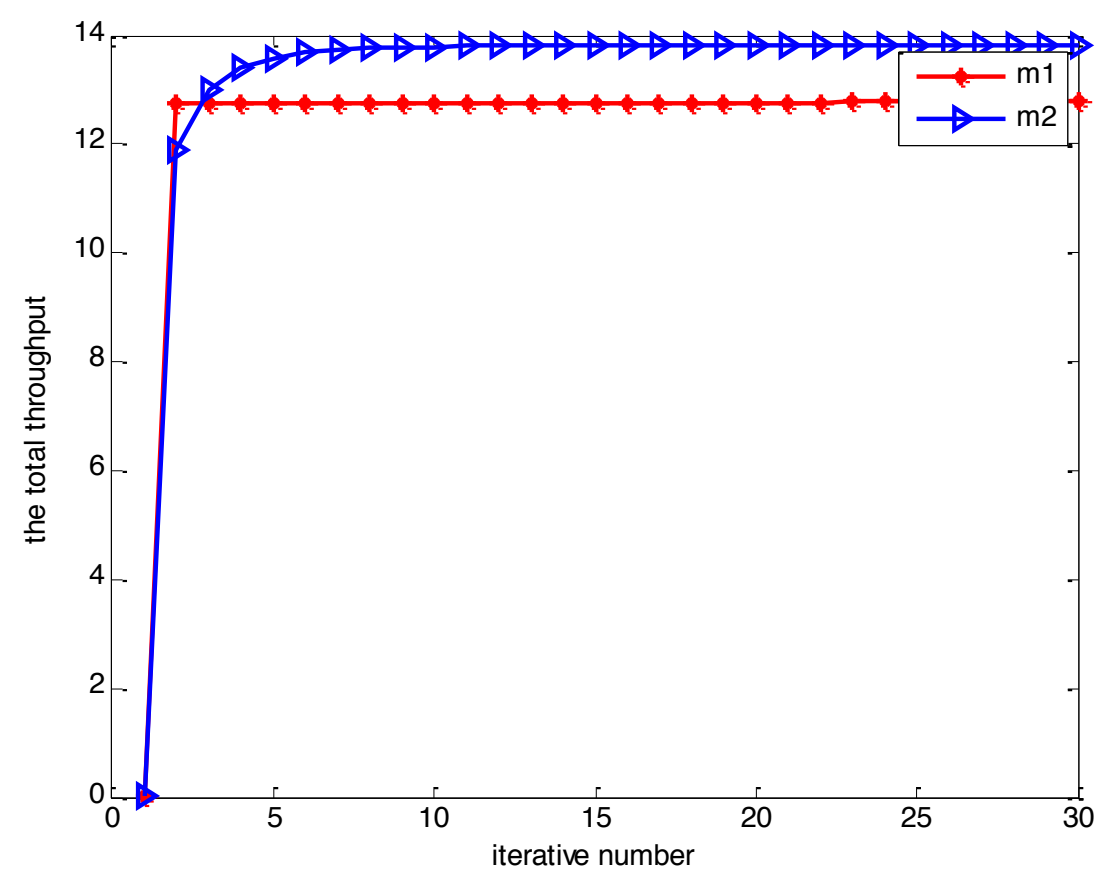

Fig. 3. The total throughput 
Paper-Distributed Power Control Algorithm in Orthogonal Frequency Division Multiple Cognitive

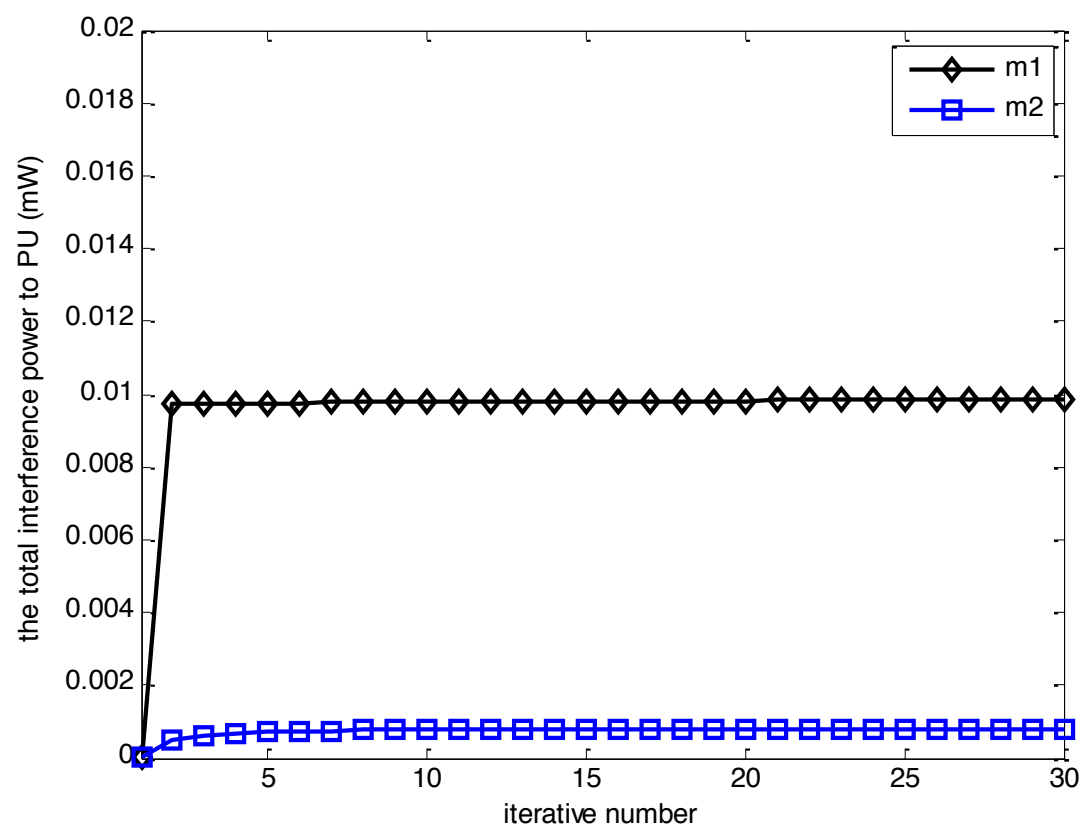

Fig. 4. The total interference to PU

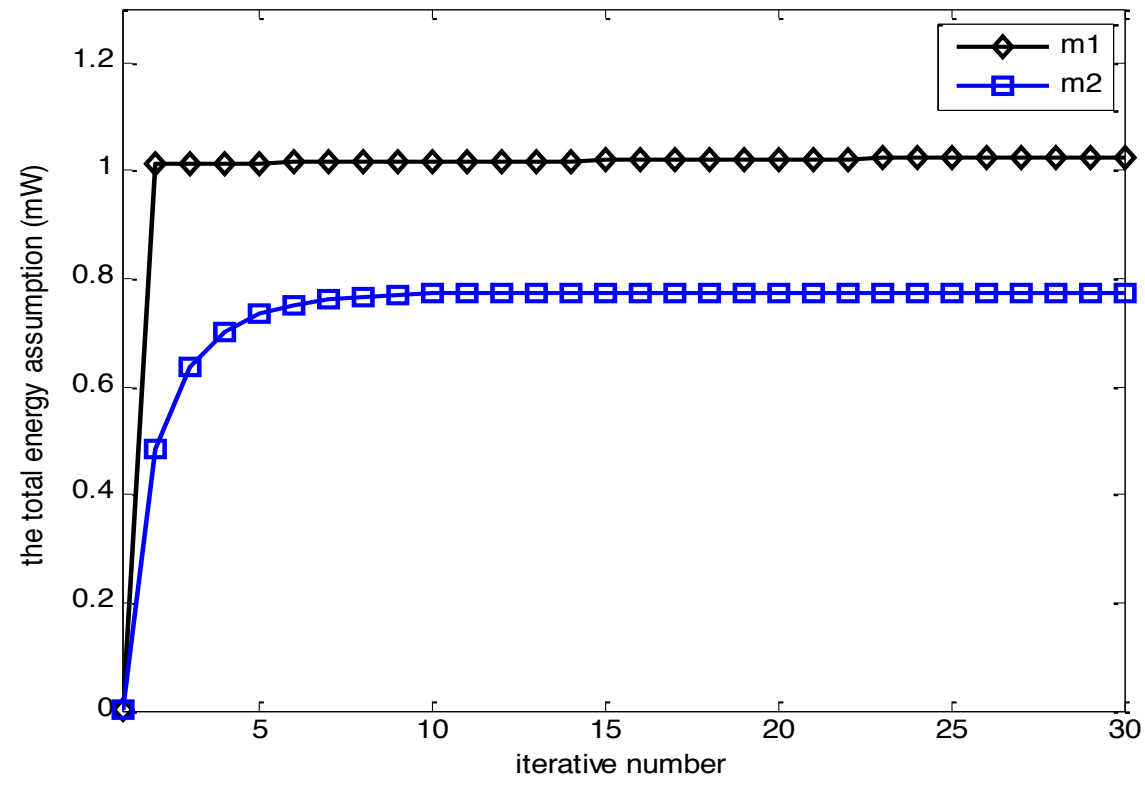

Fig. 5. The total energy consumption 


\section{Conclusions}

In this study, we solve the transmit data rate of each $\mathrm{SU}$ at all subcarriers problem in OFDM cognitive network in underlay scenario. Specifically, considering near-far effect and three major constraints of the minimum accepted SINR, the maximum transmit power of each SU and the bearable threshold of the PU in each subcarrier, a distributed power control algorithm $(\mathrm{m} 2)$ is proposed based on convex optimization theory. Finally, simulation results demonstrate the performances of the proposed algorithm and prove the performances in $\mathrm{m} 2$ is superior to $\mathrm{m} 1$ (geometric programming) in fading channel.

\section{Acknowledgment}

This work is supported in part by the National Natural Science Foundation of China under grant no. 61571209 and 61501059, Natural Science Foundation of Jilin (Grant No. 20180101336JC), scientific research fund of Jilin Provincial Education Department (Grant No. 2016134), outstanding youth fund of Jilin City Science and Technology Bureau (Grant No. 20166019), scientific research project of Jilin Institute of Chemical Technology (Grant No. 2015128(D)), and major scientific research project of Jilin Institute of Chemical Technology (Grant No. 2015010).

\section{$7 \quad$ References}

[1] Liang Y C, Chen K C, Li G Y, et al. Cognitive radio networking and communications: An overview[J]. IEEE Transactions on Vehicular Technology, 2011, 60(7): 3386-3407. https://doi.org/10.1109/TVT.2011.2158673

[2] Mitola J, Maguire Jr G Q. Cognitive radio: making software radios more personal [J]. IEEE Personal Communications, 1999, 6(4):13-18. https://doi.org/10.1109/98.788210

[3] S. Haykin, Cognitive radio: Brain-empowered wireless communications, IEEE J. Sel. Areas Commun 2005,23(2):201-220. https://doi.org/10.1109/JSAC.2004.839380

[4] Yongjun Xu, Xiaohui Zhao, Yingchang Liang. Robust power control and beamforming in cognitive radio networks: a survey[J]. IEEE Communications Surveys and Tutorials, 2015, 17(4): 1-1. https://doi.org/10.1109/COMST.2015.2425040

[5] Srinivasa, S. and Jafar, S.A., Cognitive radios for dynamic spectrum access the throughput potential of cognitive radio: A theoretical perspective, IEEE Communications zine ,2007, 45(5): 73-79. https://doi.org/10.1109/MCOM.2007.358852

[6] Taskeen Nadkar, Vinay Thumar, G.P.S. Tej, S.N Merchant and U.B. Desai, Distributed power allocation for secondary users in a cognitive radio scenario, IEEE Transactions on Wireless Communications 11(4) (2012) 1576-1586 https://doi.org/10.1109/TWC.2012. $\underline{020712.111502}$

[7] Lin X, Rasool S B. Distributed and provably efficient algorithms for joint channelassignment, scheduling, and routing in multichannel ad hoc wireless networks[J]. , IEEE/ACM Transactions on Networking, 2009, 17(6): 1874-1887. https://doi.org/10.1109/ TNET.2009.2021841

[8] Sun S, Di J, Ni W. Distributed power control based on convex optimization in cognitive radio networks[C]//Wireless Communications and Signal Processing (WCSP), 2010 International Conference on. IEEE, 2010: 1-6. 
Paper-Distributed Power Control Algorithm in Orthogonal Frequency Division Multiple Cognitive ...

[9] Yang K, Xu W, Li S, et al. Distributed multicast resource allocation in OFDM-based cognitive radio networks[C]//IEEE 2013 8th International ICST Conference Communications and Networking in China (CHINACOM), on. , 2013: 57-62.

[10] Kiani S G, Gesbert D, Gjendemsjo A, et al. Distributed power allocation for interfering wireless links based on channel information partitioning[J]. IEEE Transactions on Wireless Communications, 2009, 8(6): 3004-3015. https://doi.org/10.1109/TWC.2009.080215

[11] Lingling Chen, Xiaohui Zhao, Distributed power control algorithm for cognitive radio networks based on geometric programming[J]. Journal of Information and Computational Science,2014,11(13),4747-4757. https://doi.org/10.12733/jics20104997

[12] D. P. Bertsekas, Nolinear Programming $2^{\text {nd }}$ ed, Belmont Mass: Althena Scienfific, 1999.

[13] Didem. Gozupek, F. Alagoz, Throughput and delay optimal scheduling in cognitive radio networks under interference temperature constraints[J]. Journal of Communications and Networks 2009,11(2): 148-156. https://doi.org/10.1109/JCN.2009.6391389

[14] S. Boyd and L. Vandenberghe, Convex Optimization, Cambridge University Press, 2004. https://doi.org/10.1017/CBO9780511804441

[15] LIU Xin, JIA Min and GU Xuemai, Joint optimal sensing threshold and subcarrier power allocation in wideband cognitive radio for minimising interference to primary user[J]. China Communications, 2013,10(11): 70-80. https://doi.org/10.1109/CC.2013. 6674212

[16] Didem. Gozupek, F. Alagoz. Throughput and delay optimal scheduling in cognitive radio networks under interference temperature constraints[J]. Journal of Communications and Networks. 2009,11(2): 148-156. https://doi.org/10.1109/JCN.2009.6391389

\section{Authors}

Lingling Chen received her Bachelor and PH.D degrees both in Communication Engineering from Jilin University, China, in 2004 and 2015 respectively. Currently, she is a associate professor of College of Information and Control Engineering, Jilin Institute of Chemical Technology, simultaneously she is a post-doctor in Computer science and technology from Jilin University. Her research interests are in nonlinear optimization, mobile computing and cognitive radio networks. E-mail: cll807900@ 163.com.

Qi Feng received his Bachelor degree in Control Science and Control Engineering from North China Electric Power University, China, in 2015. Currently, he is a student of College of Information and Control Engineering, Jilin Institute of Chemical Technology. His research interests are in robust control and cognitive radio networks. E-mail: 1437149994@qq.com.

Xingquan Gao received his Master and PH.D degrees both in Control Engineering from Jilin University, China, in 2003 and 2006 respectively. Currently, he is a professor of College of Information and Control Engineering, Jilin Institute of Chemical Technology. His research interests are in nonlinear optimization, robust optimization and cognitive radio networks. E-mail: $113315903 @$ qq.com.

Huipeng Wang will receive his Bachelor degree in Electronic Information Engineering from Jilin Institute of Chemical Technology, China, in 2019. Currently, he is a student of College of Information and Control Engineering, Jilin Institute of Chemical Technology. His research interests are in tensor analysis and cognitive radio networks. E-mail: 1300168342@qq.com 
Jiwen Liang will receive his Bachelor degree in Electronic Information Engineering from Jilin Institute of Chemical Technology, China, in 2019. Currently, he is a student of College of Information and Control Engineering, Jilin Institute of Chemical Technology. His research interests are in robust optimization and cognitive radio networks. E-mail: 977967520@qq.com

Article submitted 11 February 2018. Final acceptance 05 March 2018. Final version published as submitted by the authors. 